\title{
Asymmetric Ossification
}

National Cancer Institute

\section{Source}

National Cancer Institute. Asymmetric Ossification. NCI Thesaurus. Code C124496.

A finding of a lack of symmetry between two or more bilateral aligned primary ossific nuclei (ossification centers), typically indicated by greater stain uptake in one or more primary ossification centers as compared to others. 